\title{
MS25-P108 LATE | On the Design of a Dedicated Electron Diffractometer
}

Hovestreydt, Eric (ELDICO Scientific AG, Villigen, CH); Santiso-Quinones, Gustavo (ELDICO Scientific, Villigen, CH); Steinfeld, Gunther (ELDICO Scientific, Villigen, $\mathrm{CH}$ )

Electron Diffraction (ED) as such has been around since the early days of Electron Microscopy. Since Transmission Electron Microscopes (TEMs) are available with accelerating powers of 200 to $300 \mathrm{kV}$ and 2D detectors have become fast enough, Electron Crystallography really took off.

So far, ED has been done in TEMs that are modified, resulting in challenging experiments and limited datasets, yet, structures could be obtained from samples in the range of merely tens of nanometers, that were unsolvable with either conventional or even synchrotron X-ray radiation.

For some reason, no dedicated Electron Diffractometer has been available commercially so far. Data quality would greatly benefit from a TEM setup that focuses on the diffraction capability over imaging and allowing for faster and more complete datasets.

We will present a possible Electron Diffractometer design for Electron Crystallography from the point-of-view of Xray Crystallography and indicate improvements over present TEM-based as well as X-ray instruments. It is envisioned, that preliminary results will also be presented.

[1] www.eldico-scientific.com 